\title{
SCIENTIFIC REPORTS

\section{OPEN One Step Fabrication of Highly Absorptive and Surface Enhanced Raman Scattering (SERS) Silver Nano-trees on Silicon Substrate}

Received: 4 June 2019

Published online: 19 September 2019

\section{Sara Abdel Razek, Ahmed B. Ayoub \& Mohamed A. Swillam (D) \\ Silver Nano-trees (AgNTs) were synthesized by one-step electroless method with different densities via water or ethylene glycol (EG) on silicon substrate in one minute. The density of AgNTs is controlled by changing the concentration of silver nitrate in etchant solution. The absorption of NTs fabricated via EG is higher than absorption of NTs without EG. The AgNTs are employed as substrates for surface- enhanced Raman scattering (SERS) and exhibit high sensitivity. The silver Nano-trees fabricated via ethylene glycol (AgNTs-EG) enhances the Raman spectrum of pyridine (Py) with higher enhancement factor. Moreover, the SERS-active substrates prepared by using EG were able to detect Pyridine with concentration as low as $0.005 \mathrm{mM}$, the ones fabricated by water could only detect Pyridine at concentration of $0.2 \mathrm{mM}$.}

Recently, nanostructures of noble metals have been quickly developed into promising applications such as catalysis, electronic systems, sensing, and surface enhanced Raman scattering (SERS) $)^{1,2}$ due to their unique electrical, optical and catalytic properties that are different from the bulk metals. The metal nanostructures have intrinsic properties that can be tuned by controlling their size, shape and crystallinity as shown $\mathrm{in}^{3-8}$. SERS is sensitive to the interface with metal nanoparticles ${ }^{9,10}$. The size and shape of the nanoparticles have strong effect on the strength of the Raman enhancement ${ }^{11}$. Nano-particles optimum size is wavelength-dependent, and it is in a range from about 50 to $100 \mathrm{~nm}$ from the visible to the NIR range ${ }^{12}$. The metal Tree-like nanostructure is appropriate for enhancing the SERS effect ${ }^{13}$. One of the enhancement mechanisms which is known as electromagnetic enhancement of the silver film is correlated with the excitation of surface plasmons through the Nano-trees. Excited surface plasmons on the tip of Nano-trees are associated with collective electron oscillations, which create a localized electro- Magnetic field (EM). Another type of the enhancement mechanisms (denoted as chemical enhancement) is correlated with the chemical adsorption absorption of Pyridine (Py) on silver ${ }^{14}$.

Among different metallic nanostructures, silver nanostructures have gained interest in different applications due to its low toxicity. It supports surface plasmons (SPs) in the visible and near-infrared regions (NIR), which leads to fabrication of more effective SERS active substrates ${ }^{15,16}$. Their UV/VIS spectrum, known as the surface plasmon absorption band (SPAB), is generated by the oscillation of the conduction electrons in the particles as a result of the incident light. The SPAB is affected by the size ${ }^{17-19}$, shape ${ }^{20}$, poly-dispersity ${ }^{21}$, aggregation ${ }^{22,23}$, surrounding medium, and geometric arrangements of metal nanoparticles ${ }^{21}$. Silver also has the highest thermal and electrical conductivities among all metals ${ }^{24,25}$. Therefore, silver nanoparticles have found great areas of applicability owing to their optical, thermal, and electrical properties. One of the most common nanostructures is silver dendrites that called silver Nano-trees (AgNTs), which consist of different generations of branches ${ }^{26-29}$. Multi-levels of branching structures increase the specific surface area of substrate. AgNTs may support EM coupling in the space between two adjacent branches due to coupling of the surface plasmon polaritons (SPP). Consequently, the generation of "hot spots" increases in the spacing at the end and among branches of silver. Although different silver nanostructures have high SERS properties, the surfaces are extremely sensitive to oxidation; accordingly, the time stability in SERS application is low.

Anisotropic crystal growth and diffusion-limited aggregation (DLA) effect plays important roles in the fabrication of Nano-trees ${ }^{30-35}$. As a result, the variety of the dendritic structures can be realized by balancing these two factors. There are many theoretical attempts to explain the process of growing dendrites ${ }^{36}$. Ref. ${ }^{36}$ reported 
that the anisotropy of the solid-liquid interfacial energy, $g(n \sim)$ determines the orientation selection. An oriented attachment mechanism was improved to explain the variety of hierarchical dendrite 0 . The competition between the kinetic and thermodynamic factors is responsible for converting the fractal pattern to Ag Nano-trees.

Ethylene glycol (EG) can be utilized as reducing agent to synthesize metal nanoparticles by the polyol process $^{37}$. EG can be used for producing silver nanoparticles as solvent and reducing agent to increase the stability of the obtained Ag colloid without adding costabilizers. Moreover, the semiconducting substrate such as Si can be used for enhancing the effect of the SERS and improving the long-term stability by preventing the oxidization of silver ${ }^{38,39}$.

In this paper, we propose a simple one-minute electroless etching process to fabricate AgNTs on Si substrate by using two types of etchant solutions with different concentrations of silver nitrate at room temperature. The AgNTs were fabricated using a large-sized Si substrate to increase ability of detection of pyridine. High absorption is achieved using etchant solution. The optical properties of NTs have been investigated.

\section{Results and Discussion}

Figure 1 shows the SEM images of the obtained AgNTs by using the two different aqueous solutions. The morphology of the fabricated AgNTs was examined using a Zeiss Lro Supra 55 field emission scanning electron microscope (FESEM) to show the effect of using ethylene glycol in the etchant solution. The growth speed of AgNTs became even faster with the increase of concentration of silver nitrate, because AgNT can capture silver ions more rapidly in shorter mean free path through the growth process and reduce it into silver ${ }^{38}$. The number of the fabricated AgNTs increases with increasing the concentration of silver nitrate in the two solutions and have long central backbone. The existence of ethylene glycol in second solution generates colloidal Nano-trees. The energy dispersive $\mathrm{x}$-ray (EDX) spectra of samples in two groups have been measured and shown in Fig. 2a, b to confirm the existence of silver. As shown in Fig. 2, it indicates the presence of Ag and Si substrate. Figure 2c shows the relationship between the concentration of silver nitrate and the weight percentage of silver deposited on silicon for two groups. The weight percentage of silver deposited on silicon when the solution contained water only, is higher than the weight percentage of silver deposited on silicon in existence of ethylene glycol in all concentrations of $\mathrm{AgNO}_{3}$. Figure 2d shows the typical X-ray diffraction (XRD) patterns of fabricated AgNTs by two different concentrations of silver nitrate $(5,15 \mathrm{mM})$ in two solutions. The XRD analysis was performed using $\mathrm{Cu}$ $\mathrm{K} \alpha 1$ radiation source $\left(\lambda=1.5406 \mathrm{~A}^{\circ}\right)$. The XRD patterns show sharp peaks that are indexed to (111), (200), (220), (311) diffraction peaks of the FCC Ag, and Si (111) diffraction peak comes from the Si substrate. The intensities of silicon peak of the first (AgNTs-wt1) and third (AgNTs-EG1) samples were higher than the second (AgNTs-wt5) and fourth (AgNTs-EG5) samples due to the low concentration of deposited silver. Moreover, the intensities of Ag peaks with all orientations, of the second (AgNTs-wt5) and fourth (AgNTs-EG5) samples were greater than the first (AgNTs-wt1) and third (AgNTs-EG1) samples due to increasing of silver.

\section{Discussion}

Optical properties. The absorption spectra as shown in (Fig. 3) was measured using a Perkin-Elmer-Lambda UV-NIR and visible spectrophotometer with solid-sample holder for reflectance measurements and a universal reflectance unit where the reflection " $R$ " is measured and then the absorption is measured as " $A=1-R$ ". The absorption of AgNTs fabricated without ethylene glycol increases with increasing the concentration of $\mathrm{AgNO}_{3}$ in the first three concentrations, then the absorption decreases with continuous increase of $\mathrm{AgNO}_{3}$ due to increase of density of the fabricated AgNTs. The increased density of AgNTs enhances the scattering of light in the last two samples.

On the other hand, the absorption of the AgNTs synthesized in the presence of ethylene glycol increases with increasing the concentration of silver nitrate. The ethylene glycol here worked as reducing agent to increase the stability of the obtained silver collides. Generally, the absorption of silicon, throughout the entire wavelength range, increased by adding AgNTs of different densities due to the multiple reflection of light through AgNTs, i.e. trapping light. The absorption of the AgNTs-EG is larger than absorption of AgNTs-wt in all various concentrations of $\mathrm{AgNO}_{3}$ as shown in Fig. 3 due to the high refractive index of ethylene glycol ${ }^{39}$. In Ref. ${ }^{40}$, the same study was performed for silver nanoparticles (AgNPs). The AgNPs experienced higher absorption when immersed in ethylene glycol not water, since the higher refractive index of the ethylene resulted in higher extinction cross section (an indication on absorption) $)^{41}$ through Eq. (1)

$$
C_{e x t}=9\left[\frac{\omega}{c} \varepsilon_{m}^{3 / 2} \frac{\varepsilon_{i}}{\left(\varepsilon_{r}+2 \varepsilon_{m}\right)^{2}+\varepsilon_{i}^{2}}\right]
$$

where $\varepsilon_{m}$ is the permittivity of the surrounding medium, $\varepsilon_{i}$ is the imaginary part of the metal permittivity, and $\varepsilon_{r}$ is the real part of the metal permittivity. The increase in the extinction cross section results in higher absorption coefficient. Although AgNTs differ from AgNPs in their geometrical structure, however, the general rules of scattering and absorption still hold and similar trends in the absorption properties are observed with different values. For example, when the silver nitrate concentration was $5 \mathrm{mM}$, the maximum absorption of AgNTs-wt1 was 0.9904 compared with AgNTs-EG1 that was 0.995 at wavelength $370 \mathrm{~nm}$, which is a small difference 0.0046 as shown in Fig. 3a. The difference between the two absorption patterns increased to 0.13434 at wavelength of $800 \mathrm{~nm}$. The absorption increasing in AgNTs-wt was continued till the concentration of $\mathrm{AgNO}_{3}$ was $10 \mathrm{mM}$ to reach its maximum absorption 0.9961 at $370 \mathrm{~nm}$, then started to decrease to reach 0.9918 for $15 \mathrm{mM}$ of $\mathrm{AgNO}_{3}$. In contrast, the absorption of AgNTs-EG continued to increase with increasing the $\mathrm{AgNO}_{3}$ concentration to reach its maximum $\approx 0.997$ for $15 \mathrm{mM}$ of $\mathrm{AgNO}_{3}$ at wavelength $370 \mathrm{~nm}$. Moreover, the absorption of AgNTs-EG5 was larger than absorption of AgNTs-wt5 by $(\approx 0.005)$ in wavelength $370 \mathrm{~nm}$ and the difference was increased in higher wavelength to be 0.0213 at wavelength of $800 \mathrm{~nm}$ as shown in Fig. 3b. The increasing and decreasing in 


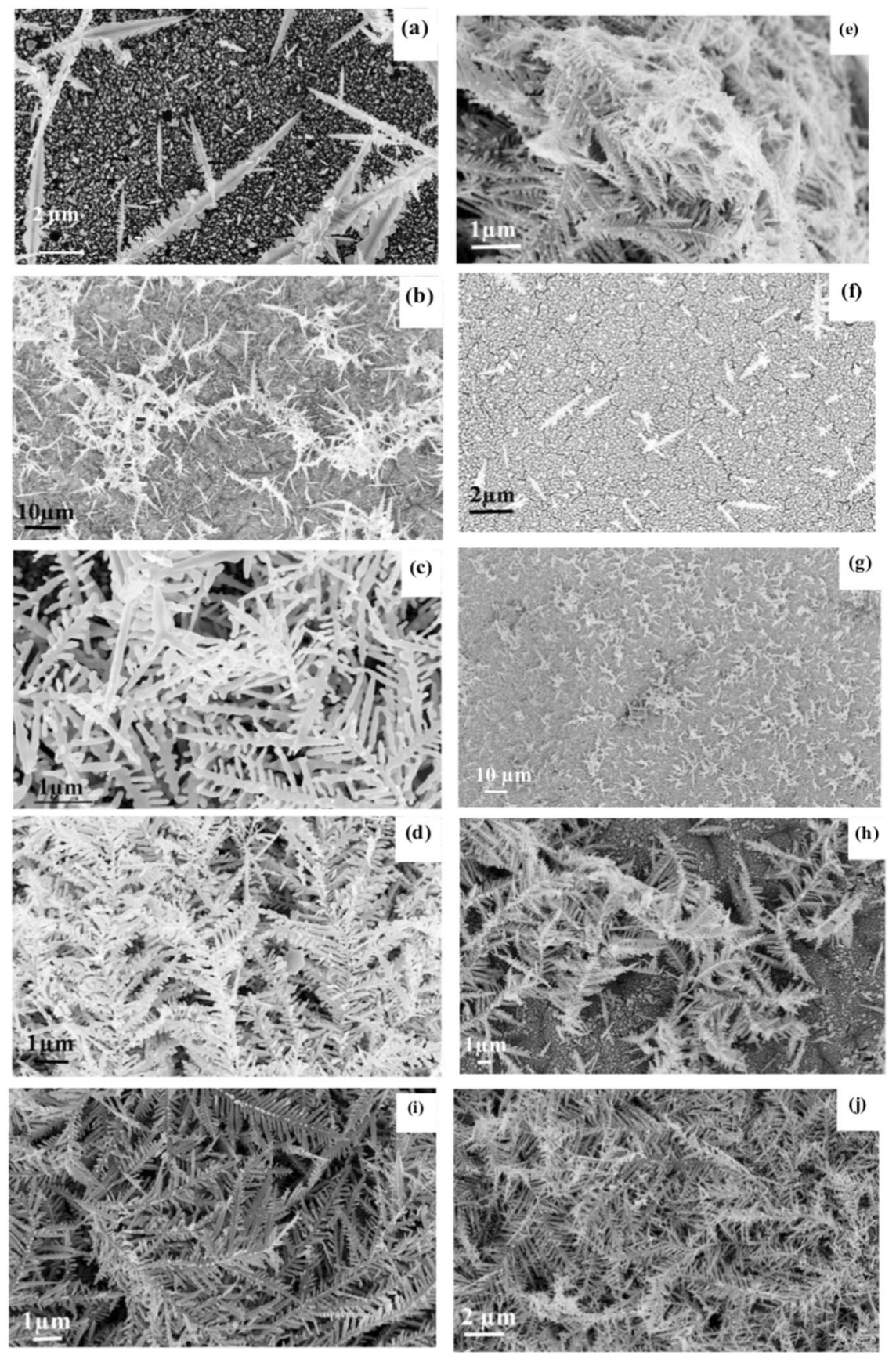

Figure 1. FESEM of the fabricated Ag Nano-trees in two different solutions: group A (without ethylene glycol) when the concentration of silver nitrate was (a) $5 \mathrm{mM}$, (b) $7.5 \mathrm{mM},(\mathbf{c}) 10 \mathrm{mM}$, (d) $12.5 \mathrm{mM}$, and (e) $15 \mathrm{mM}$, group B (with ethylene glycol) when the concentration of silver nitrate was (f) $5 \mathrm{mM},(\mathbf{g}) 7.5 \mathrm{mM},(\mathbf{h}) 10 \mathrm{mM}$, (i) $12.5 \mathrm{mM}$, and (j) $15 \mathrm{mM}$.

absorbance of AgNTs fabricated without EG depends on the density of the fabricated AgNTs. When the density of the AgNTs was small, the absorption is enhanced by increasing the trapping of light through the trees till light trapping process is transformed into light scattering in high densities of Ag. Figure $3 \mathrm{c}$ presents a maximum absorption of silver Nano-trees fabricated in water and ethylene glycol solutions as a function of $\mathrm{AgNO}_{3}$ concentration. Moreover, there is a small red shift in wavelength of absorption of AgNTs-EG that is around $10 \mathrm{~nm}$ due to high refractive index of ethylene glycol ${ }^{39}$. This shift is due to the resonance peak shift in the polarizability. Referring to $\mathrm{NPs}^{41}$, polarizability is given by

$$
\alpha=4 \pi a^{3} \frac{\varepsilon(\omega)-\varepsilon_{m}}{\left(\varepsilon(\omega)+2 \varepsilon_{m}\right)^{2}}
$$



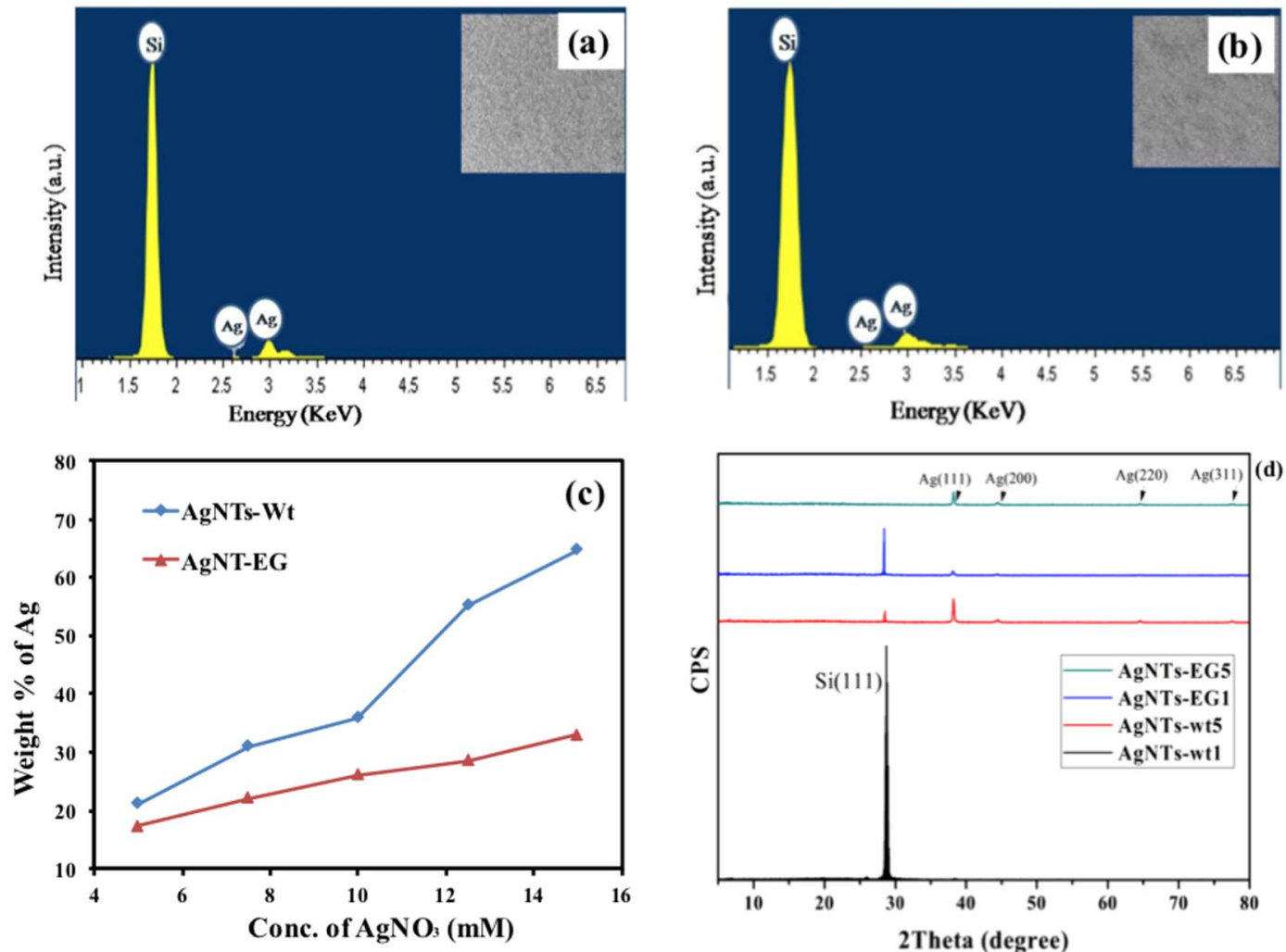

Figure 2. EDX spectra of (a) AgNT-wt1, (b) AgNT-EG1, and (c) relationship between weight \% of Ag and concentration of $\mathrm{AgNO}_{3}$ for two types of fabricated NTs, and (d) XRD patterns of AgNTs concentration. Although the (AgNTs-wt5) and the (AgNTs-EG5) samples experience Ag peaks in different orientation, it could be observed from the figure that the peak along the (111) axis is the dominating one from the XRD patterns. Hence, the long central branches obviously point to the preferred growth direction along the (111) axis. Each Nano-tree has a sharp secondary branch that has few secondary branches.

From Eq. (2), the polarizability is inversely proportional with $\left(\varepsilon(\omega)+2 \varepsilon_{m}\right)^{2}$, where $\varepsilon(\omega)$ is the permittivity of the metal and $\varepsilon_{m}$ is the permittivity of the surrounding medium. From this relation, it is obvious that the higher medium permittivity leads to resonance at longer wavelength, and hence the absorption resonant wavelength experiences a red shift in wavelength. Therefore, ethylene glycol results in a red shift in the absorption resonant wavelength as compared to water owing to its higher refractive index.

Raman spectroscopy. Raman spectroscopy provides information on vibrational, molecular, and electronic levels. Raman scattering is one of the most powerful techniques utilized to identify chemical and biological samples through the detection of the characteristic oscillations of the molecules contained in samples. Through this phenomenon, molecules adsorbed onto a metal surface under specific conditions show a large interaction cross-section for the Raman effect, as a result, the Raman signal of these molecules is amplified by several orders of magnitude ${ }^{42-44}$. To find out the detection limit for pyridine (Py) on AgNTs fabricated on silicon substrate by electroless method, we measured the Raman spectra of Py applied to 8 samples. Pyridine was chosen as a test compound for investigating the application of AgNT structure to detect the trace of toxic organic compounds.

Raman measurements were performed by using Micro Raman setup (ProRaman-L Analyzer PRO-L-5B1S) with an excitation laser beam wavelength of $532 \mathrm{~nm}$ with maximum power of $50 \mathrm{~mW}$ with an accumulation time was varied between 60 and 30 seconds for each set of experiments. Figure $4 a$,b, with an accumulation time of 60 seconds, shows the SERS spectra of Py with concentration of $0.2 \mathrm{mM}$ for 8 different samples. The combination of Fig. 1 with Fig. $4 \mathrm{~b}$ clearly shows the correspondence between the morphology of AgNTs, and the amplification of a Raman spectrum as expected. This fact can be explained on the basis of increasing the number of "hot spots". In the SERS substrates fabricated by using EG, because of the better divergence of tree branches, the number of sharp 'lighting' tips as well as of 'nano-gaps' increased. Thus the number of 'hot spot' to amplify the Raman signal has become much greater. Consequently, the SERS enhancement of AgNTs-EG is significantly higher compared with AgNTs-wt ${ }^{45}$.

First peak appeared around $520 \mathrm{~cm}^{-1}$ in all 8 samples. The detection of Py in all AgNT-EG is shown clearly in Fig. 4b. Two strong Raman band at about $\sim 1004$ and $1032 \mathrm{~cm}^{-1}$ were clearly observed in the spectrum at 7 samples. Sample 1 that has the lowest concentration of silver and fabricated without EG did not have any peak of Py. The peaks of Py are enhanced with increasing the silver concentration with using EG in growth process. To find out the detection limit for Py of AgNTs-EG, we have used AgNTs-EG5 to record the Raman spectra of Py with different concentrations, including $0.005,0.01,0.1,0.15$ and $0.2 \mathrm{mM}$ at peak $\sim 1004 \mathrm{~cm}^{-1}$ at an accumulation 

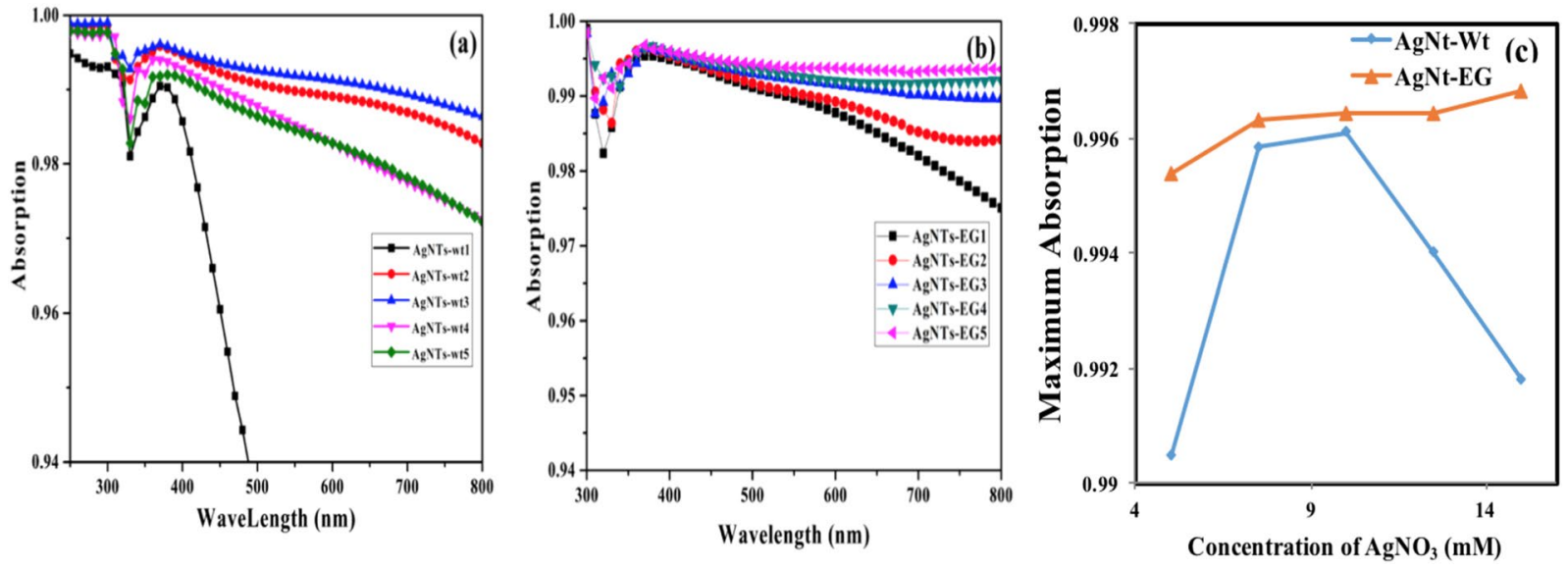

Figure 3. Absorption of fabricated AgNTs with different concentrations of $\mathrm{AgNO}_{3}$ (a) when the etchant solution contained water only, (b) when the etchant solution contained ethylene glycol and (c) relationship between maximum absorption of all fabricated NTs and concentration of $\mathrm{AgNO}_{3}$ at wavelength $370 \mathrm{~nm}$ (due to maximum absorption at this wavelength).
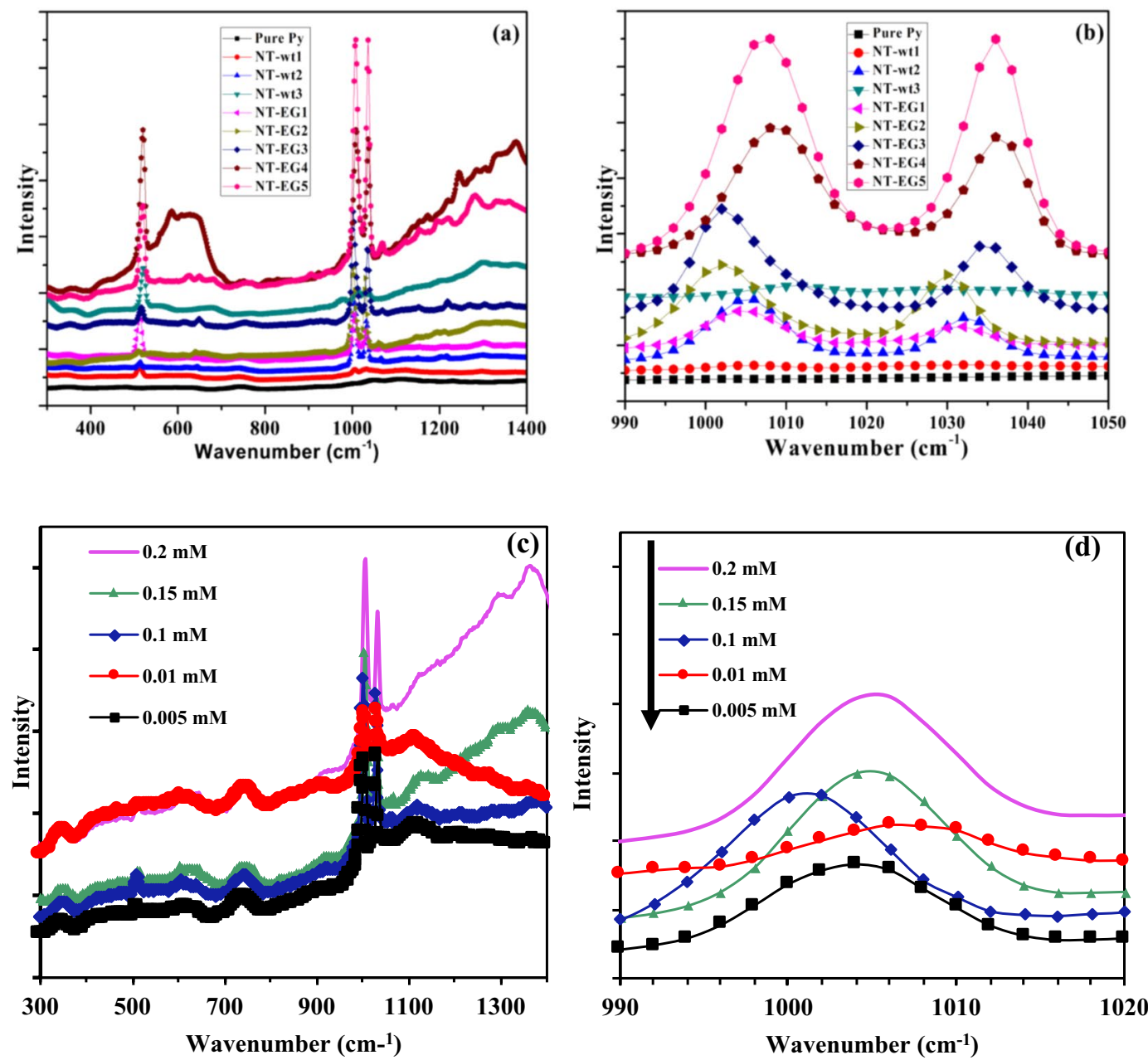

Figure 4. (a,b) Raman spectra of undiluted pure pyridine and fabricated AgNTs in different etchant solutions and concentration of $\mathrm{AgNO}_{3}$ fabricated on silicon substrate, and (c) and (d) Raman spectra of Py with different concentrations dripped on AgNTs-EG5. 
time of 30 seconds. The signal intensity increased concomitantly with an increase in Py concentration as shown in Fig. 4c,d. We calculate the enhancement factor (EF) as the ratio between the intensity of the SERS to that of pure pyridine. The reason we used this way of calculation is that other techniques for the calculation of the EF assume nanoparticles (nano-spheres) with specific particle diameter which is not the case in the manuscript since we are studying nanotrees. Using this definition of the enhancement factor as follows:

$$
E F=\frac{I_{\text {sers }}}{I_{\text {pure }}},
$$

where I is the intensity at a certain wavenumber. Using this equation we have an enhancement factor of 16 at wavenumber $1008 \mathrm{~cm}^{-1}$ for AgNT-EG5 as shown in Fig. 4a. Although the EF reported in literature are in orders of powers of 10, however, those EFs do take into account the geometrical parameters of the sample as explained above. Otherwise, the ratio of the intensities would be in the range of 5 to 10 as reported in literature ${ }^{46}$. As shown in Fig. 4c,d, EF is around 2.5. However, such EF value can be enhanced for higher accumulation time.

\section{Experimental Method}

The n-type (111) oriented mono-crystalline $\mathrm{Si}$ wafer with a resistivity of $0.002-0.0045 \Omega \mathrm{cm}$ was used to template the formation of Ag Nano-trees. The Si samples were cleaned as reported in our previous work. After the cleaning procedures, Si samples were divided to two groups, first group immersed in aqueous solution A that contained $10 \% \mathrm{HF}$ and different concentrations of silver nitrate $(5,7.5,10,12.5,15) \mathrm{mM}$ for 1 minute with stirring at room temperature, to fabricate samples AgNT-wt1, AgNTs-wt2, AgNTs-wt3, AgNTs-wt4 and AgNTs-wt5, respectively. The second group of Si samples was immersed in aqueous solution B that contained $10 \% \mathrm{HF}, 10 \%$ of ethylene glycol and different concentrations of silver nitrate $(5,7.5,10,12.5$, and 15$) \mathrm{mM}$ for 1 minute also with stirring at room temperature, to fabricate samples AgNT-EG1, AgNT-EG2, AgNT-EG3, AgNT-EG4, and AgNT-EG5 respectively. The above steps were performed five times for all samples to make sure that the same results could be obtained every time with following the same steps. For SERS measurements, the pyridine with different concentrations was added drop wise on the substrate surface.

\section{Conclusions}

The AgNTs deposited on silicon have been fabricated via one step electroless method in one minute. The density of AgNTs could be controlled by changing the concentration of $\mathrm{AgNO}_{3}$ through etching process. The absorption of AgNTs-EG is higher than the absorption of AgNT-wt in all concentrations because of higher refractive index of ethylene glycol. Moreover, AgNTs-EG have high enhancement factor of SERS. The AgNTs-EG can detect Py with concentration as low as $0.005 \mathrm{mM}$, while AgNTs-wt can only detect Py with concentration as low as $0.2 \mathrm{mM}$.

\section{References}

1. Aroca, R. \& Rodriguez-Llorente, S. Surface-enhanced vibrational spectroscopy. Journal of Molecular Structure 408-409, 17-22, https://doi.org/10.1016/S0022-2860(96)09489-6 (1997).

2. Bjerneld, E. J., Svedberg, F. \& Käll, M. Laser-Induced Growth and Deposition of Noble-Metal Nanoparticles for Surface-Enhanced Raman Scattering. Nano Letters 3, 593-596, https://doi.org/10.1021/nl034034r (2003).

3. Choy, J.-H. Preface. Journal of Physics and Chemistry of Solids 69, 1039, https://doi.org/10.1016/j.jpcs.2007.11.006 (2008).

4. Chumanov, G., Sokolov, K. \& Cotton, T. M. Unusual Extinction Spectra of Nanometer-Sized Silver Particles Arranged in TwoDimensional Arrays. The Journal of Physical Chemistry 100, 5166-5168, https://doi.org/10.1021/jp9536716 (1996).

5. Cowcher, D. P., Xu, Y. \& Goodacre, R. Portable, Quantitative Detection of Bacillus Bacterial Spores Using Surface-Enhanced Raman Scattering. Analytical Chemistry 85, 3297-3302, https://doi.org/10.1021/ac303657k (2013).

6. Dao, T. C., Luong, T. Q. N., Cao, T. A., Kieu, N. M. \& Le, V. V. Application of silver nanodendrites deposited on silicon in SAB. Advances in Natural. Sciences: Nanoscience and Nanotechnology 7, 015007, https://doi.org/10.1088/2043-6262/7/1/015007 (2016).

7. Gajaraj, S., Fan, C., Lin, M. \& Hu, Z. Quantitative detection of nitrate in water and wastewater by surface-enhanced Raman spectroscopy. Environmental Monitoring and Assessment 185, 5673-5681, https://doi.org/10.1007/s10661-012-2975-4 (2013).

8. Haxhimali, T., Karma, A., Gonzales, F. \& Rappaz, M. Orientation selection in dendritic evolution. Nature Materials 5, 660-664, https://doi.org/10.1038/nmat1693 (2006).

9. Cai, W., Hofmeister, H. \& Rainer, T. Surface effect on the size evolution of surface plasmon resonances of Ag and Au nanoparticles dispersed within mesoporous silica. Physica E: Low-dimensional Systems and Nanostructures 11, 339-344, https://doi.org/10.1016/ S1386-9477(01)00171-0 (2001).

10. Callegari, A., Tonti, D. \& Chergui, M. Photochemically Grown Silver Nanoparticles with Wavelength-Controlled Size and Shape. Nano Letters 3, 1565-1568, https://doi.org/10.1021/nl034757a (2003).

11. Huang, H. H. et al. Photochemical Formation of Silver Nanoparticles in Poly(N-vinylpyrrolidone). Langmuir 12, 909-912, https:// doi.org/10.1021/la950435d (1996).

12. Hudson, S. D. \& Chumanov, G. Bioanalytical applications of SERS (surface-enhanced Raman spectroscopy). Analytical and Bioanalytical Chemistry 394, 679-686, https://doi.org/10.1007/s00216-009-2756-2 (2009).

13. Hwang, C.-B. et al. Synthesis, Characterization, and Highly Efficient Catalytic Reactivity of Suspended Palladium Nanoparticles. Journal of Catalysis 195, 336-341, https://doi.org/10.1006/jcat.2000.2992 (2000).

14. Jing, C. \& Fang, Y. Simple method for electrochemical preparation of silver dendrites used as active and stable SERS substrate. Journal of Colloid and Interface Science 314, 46-51, https://doi.org/10.1016/j.jcis.2007.05.041 (2007).

15. Kelly, K. L., Coronado, E., Zhao, L. L. \& Schatz, G. C. The Optical Properties of Metal Nanoparticles: The Influence of Size, Shape, and Dielectric Environment. The Journal of Physical Chemistry B 107, 668-677, https://doi.org/10.1021/jp026731y (2003).

16. Le R, E. C. \& Etchegoin, P. G. In Principles of Surface-Enhanced Raman Spectroscopy (eds Eric C. Le Ru \& Pablo G. Etchegoin) 1-27 (Elsevier, 2009).

17. Link, S. \& El-Sayed, M. A. Spectral Properties and Relaxation Dynamics of Surface Plasmon Electronic Oscillations in Gold and Silver Nanodots and Nanorods. The Journal of Physical Chemistry B 103, 8410-8426, https://doi.org/10.1021/jp9917648 (1999).

18. Mafuné, F., Kohno, J.-y., Takeda, Y., Kondow, T. \& Sawabe, H. Structure and Stability of Silver Nanoparticles in Aqueous Solution Produced by Laser Ablation. The Journal of Physical Chemistry B 104, 8333-8337, https://doi.org/10.1021/jp001803b (2000).

19. Maier, S. A. In Plasmonics: Fundamentals and Applications (ed Stefan A. Maier) 65-88 (Springer US, 2007). 
20. Mandal, S. et al. Studies on the Reversible Aggregation of Cysteine-Capped Colloidal Silver Particles Interconnected via Hydrogen Bonds. Langmuir 17, 6262-6268, https://doi.org/10.1021/la010536d (2001).

21. Manna, A., Imae, T., Iida, M. \& Hisamatsu, N. Formation of Silver Nanoparticles from a N-Hexadecylethylenediamine Silver Nitrate Complex. Langmuir 17, 6000-6004, https://doi.org/10.1021/la010389j (2001).

22. Müller, H., Opitz, C. \& Skala, L. The highly dispersed metal state - physical and chemical properties. Journal of Molecular Catalysis 54, 389-405, https://doi.org/10.1016/0304-5102(89)80154-3 (1989).

23. Narayanan, R. \& El-Sayed, M. A. Effect of Catalysis on the Stability of Metallic Nanoparticles: Suzuki Reaction Catalyzed by PVPPalladium Nanoparticles. Journal of the American Chemical Society 125, 8340-8347, https://doi.org/10.1021/ja035044x (2003).

24. Pastoriza-Santos, I. \& Liz-Marzán, L. M. Formation of PVP-Protected Metal Nanoparticles in DMF. Langmuir 18, 2888-2894, https://doi.org/10.1021/la015578g (2002).

25. Peng, K., Yan, Y., Gao, S. \& Zhu, J. Dendrite-Assisted Growth of Silicon Nanowires in Electroless Metal Deposition. Advanced Functional Materials 13, 127-132, https://doi.org/10.1002/adfm.200390018 (2003).

26. Qiu, T., Wu, X. L., Mei, Y. F., Chu, P. K. \& Siu, G. G. Self-organized synthesis of silver dendritic nanostructures via an electroless metal deposition method. Applied Physics A 81, 669-671, https://doi.org/10.1007/s00339-005-3263-8 (2005).

27. Roucoux, A., Schulz, J. \& Patin, H. Reduced Transition Metal Colloids: A Novel Family of Reusable Catalysts? Chemical Reviews 102,3757-3778, https://doi.org/10.1021/cr010350j (2002)

28. Slistan-Grijalva, A. et al. Classical theoretical characterization of the surface plasmon absorption band for silver spherical nanoparticles suspended in water and ethylene glycol. Physica E: Low-dimensional Systems and Nanostructures 27, 104-112, https:// doi.org/10.1016/j.physe.2004.10.014 (2005).

29. Sun, Y. \& Xia, Y. Shape-Controlled Synthesis of Gold and Silver Nanoparticles. Science 298, 2176, https://doi.org/10.1126/ science.1077229 (2002).

30. Vendamani, V. S., Nageswara Rao, S. V. S., Venugopal Rao, S., Kanjilal, D. \& Pathak, A. P. Three-dimensional hybrid silicon nanostructures for surface enhanced Raman spectroscopy based molecular detection. Journal of Applied Physics 123, 014301, https://doi.org/10.1063/1.5000994 (2018).

31. Wang, J.-P., Zhao, J.-W., Duan, X.-Y. \& Niu, J.-Y. Syntheses and Structures of One- and Two-Dimensional Organic-Inorganic Hybrid Rare Earth Derivatives Based on Monovacant Keggin-Type Polyoxotungstates. Crystal Growth \& Design 6, 507-513, https:// doi.org/10.1021/cg050433j (2006).

32. Wang, S. \& Xin, H. Fractal and Dendritic Growth of Metallic Ag Aggregated from Different Kinds of $\gamma$-Irradiated Solutions. The Journal of Physical Chemistry B 104, 5681-5685, https://doi.org/10.1021/jp000225w (2000).

33. Wang, X., Shi, W., She, G. \& Mu, L. Using Si and Ge Nanostructures as Substrates for Surface-Enhanced Raman Scattering Based on Photoinduced Charge Transfer Mechanism. Journal of the American Chemical Society 133, 16518-16523, https://doi.org/10.1021/ ja2057874 (2011)

34. Weißenbacher, N., Göbel, R. \& Kellner, R. Ag-layers on non-ferrous metals and alloys. A new substrate for surface enhanced Raman scattering (SERS). Vibrational Spectroscopy 12, 189-195, https://doi.org/10.1016/0924-2031(96)00024-0 (1996).

35. Wen, X. et al. Dendritic Nanostructures of Silver: Facile Synthesis, Structural Characterizations, and Sensing Applications. Langmuir 22, 4836-4842, https://doi.org/10.1021/la060267x (2006).

36. Cheng, C. et al. Fabrication and SERS Performance of Silver-Nanoparticle-Decorated Si/ZnO Nanotrees in Ordered Arrays. ACS Applied Materials \& Interfaces 2, 1824-1828, https://doi.org/10.1021/am100270b (2010).

37. Westcott, S. L., Oldenburg, S. J., Lee, T. R. \& Halas, N. J. Formation and Adsorption of Clusters of Gold Nanoparticles onto Functionalized Silica Nanoparticle Surfaces. Langmuir 14, 5396-5401, https://doi.org/10.1021/la980380q (1998).

38. Wiley, B., Sun, Y. \& Xia, Y. Synthesis of Silver Nanostructures with Controlled Shapes and Properties. Accounts of Chemical Research 40, 1067-1076, https://doi.org/10.1021/ar7000974 (2007).

39. Wu, D.-Y. et al. Chemical Enhancement Effects in SERS Spectra: A Quantum Chemical Study of Pyridine Interacting with Copper, Silver, Gold and Platinum Metals. The Journal of Physical Chemistry C 112, 4195-4204, https://doi.org/10.1021/jp0760962 (2008).

40. Yang, J. \& Jiang, Z. Facile fabrication of dendritic silver structures and their surface enhanced Raman spectroscopic properties. Journal of Chemical Sciences 127, 173-176, https://doi.org/10.1007/s12039-014-0763-0 (2015).

41. Ye, W. et al. Self-assembled synthesis of SERS-active silver dendrites and photoluminescence properties of a thin porous silicon layer. Electrochemistry Communications 10, 625-629, https://doi.org/10.1016/j.elecom.2008.01.040 (2008).

42. Zhao, L., Kelly, K. L. \& Schatz, G. C. The Extinction Spectra of Silver Nanoparticle Arrays: Influence of Array Structure on Plasmon Resonance Wavelength and Width. The Journal of Physical Chemistry B 107, 7343-7350, https://doi.org/10.1021/jp034235j (2003).

43. Zheng, J., Stevenson, M. S., Hikida, R. S. \& Van Patten, P. G. Influence of pH on Dendrimer-Protected Nanoparticles. The Journal of Physical Chemistry B 106, 1252-1255, https://doi.org/10.1021/jp013108p (2002).

44. Zhu, J., Liu, S., Palchik, O., Koltypin, Y. \& Gedanken, A. Shape-Controlled Synthesis of Silver Nanoparticles by Pulse Sonoelectrochemical Methods. Langmuir 16, 6396-6399, https://doi.org/10.1021/la991507u (2000).

45. Zuo, X., Liu, H. \& Liu, M. Asymmetric hydrogenation of $\alpha$-ketoesters over finely dispersed polymer-stabilized platinum clusters. Tetrahedron Letters 39, 1941-1944, https://doi.org/10.1016/S0040-4039(98)00088-4 (1998).

46. Le, R. E. C., Blackie, E., Meyer, M. \& Etchegoin, P. G. Surface Enhanced Raman Scattering Enhancement Factors: A Comprehensive Study. The Journal of Physical Chemistry C 111, 13794-13803, https://doi.org/10.1021/jp0687908 (2007).

\section{Author Contributions}

M. Swillam suggested and planned the project. S.A. conceived the basic idea and designed the experiments. A.B.A. did the Raman characterization measurements including Raman spectroscopy, and analyzed the data. S.A. contributed to writing the initial draft of the paper. A.B.A. contributed to writing the paper and revision. M. Swillam led the writing, and editing the manuscript and analyzing the results. M. Swillam supervised the entire project. All authors contributed to general discussions.

\section{Additional Information}

Competing Interests: The authors declare no competing interests.

Publisher's note Springer Nature remains neutral with regard to jurisdictional claims in published maps and institutional affiliations. 
(c) (i) Open Access This article is licensed under a Creative Commons Attribution 4.0 International License, which permits use, sharing, adaptation, distribution and reproduction in any medium or format, as long as you give appropriate credit to the original author(s) and the source, provide a link to the Creative Commons license, and indicate if changes were made. The images or other third party material in this article are included in the article's Creative Commons license, unless indicated otherwise in a credit line to the material. If material is not included in the article's Creative Commons license and your intended use is not permitted by statutory regulation or exceeds the permitted use, you will need to obtain permission directly from the copyright holder. To view a copy of this license, visit http://creativecommons.org/licenses/by/4.0/.

(C) The Author(s) 2019 